\title{
Intracellular Calcium Regulation in Canine Ventricular Myocytes: a Simulation Study
}

\author{
Estefanía Renú, José M Ferrero
}

\author{
Universitat Politècnica de València, Valencia, Spain
}

\begin{abstract}
Intracellular calcium $\left(\mathrm{Ca}_{i}\right)$ regulation in cardiomyocytes is critical because of its relationship with cell contractility and arrhythmogenesis. Pharmacological interventions and/or mutations affecting ion channel expression levels may alter ionic currents and thus $\mathrm{Ca}_{i}$ handling. Because it is difficult to conduct a systematic analysis of the effects of ionic current variations using solely experimental means, we conducted computational simulations to characterize the effects of ion channel alterations on $\mathrm{Ca}_{i}$ parameters in dog cardiomyocytes. Using the Decker-Rudy model, we simulated upregulations and down-regulations of sarcolemmal and sarcoplasmic reticulum ion channels, pumps and exchangers using values ranging from $0 \%$ to $200 \%$ of their control values. We then performed a sensitivity analysis of the effects of these variations on a set of $C a_{i}$ parameters at different pacing rates. Our results show that both the SERCA pump and the $\mathrm{Na}^{+}-\mathrm{K}^{+}$pump are key in controlling diastolic calcium $(\mathrm{CaD})$, systolic calcium $(\mathrm{CaS})$, the calcium transient duration (CaTD90) and the maximum calcium upstroke velocity (CaDotMax). Other relevant factors are the current through the L-type calcium current $\left(I_{\mathrm{CaL}}\right)$ and the sarcolemmal $\mathrm{Na}^{+}-\mathrm{Ca}^{2+}$ exchanger. At high pacing frequencies, the importance of $I_{C a L}$ is enhanced.
\end{abstract}

\section{Introduction}

Intracellular calcium handling is a key process in the electrophysiology of cardiac myocytes [1,2]. On the one hand, it determines cell contractility [1], because intracellular calcium is the main regulator of myocardial excitation-contraction coupling. On the other hand, it is intimately related to arrhythmogenesis, because calcium overload may trigger abnormal cell depolarization $[2,3]$. The dynamic changes in intracellular calcium concentration $\left(\left[\mathrm{Ca}^{2+}\right]_{\mathrm{i}}\right)$ during the action potential are complex. Upon cell depolarization at the onset of an action potential (AP), calcium enters the cell through the L-type calcium channels. This triggers a massive release of calcium from the sarcoplasmic reticulum (SR) to the cytosol through the ryanodine receptors, a process that may elevate $\left[\mathrm{Ca}^{2+}\right]_{i}$ almost an order of magnitude compared to its basal value (the diastolic calcium level). After $\left[\mathrm{Ca}^{2+}\right]_{\mathrm{i}}$ peaks (reaching its systolic level), it slowly declines due to (a) extrusion to the extracellular medium via the sodiumcalcium exchanger (NCX) in the sarcolemma, and (b) uptake to the SR via the SERCA pump [4].

In the past two decades, mathematical modelling and computational simulations have been a powerful tool used to better understand the intricate mechanisms related to cellular electrophysiology of cardiac myocytes (see [5] for review). These computational studies rely on detailed and comprehensive mathematical models of action potentials for different animal species [6-8], including human [9]. Using this models, different studies have attempted to characterize the influence of the different sarcolemmal and SR ionic currents in AP and intracellular $\mathrm{Ca}^{2+}$ preclinical biomarkers $[10,11]$, but none of them has been carried out in dog, an animal that has widely been used in the past for drug and mutation effect studies $[12,13]$.

In this study, we have used a well-known existing model of the action potential and ionic currents of a dog ventricular myocyte [7] to perform a sensitivity analysis to elucidate the ionic mechanisms that affect intracellular $\mathrm{Ca}^{2+}$ regulation.

\section{Methods}

The action potential, underlying ionic currents and dynamic changes in ionic concentrations of an isolated canine ventricular myocyte were simulated using a modified version of the Decker-Rudy model $[7,13]$, which includes a detailed formulation of calcium dynamics. A sensitivity analysis was performed by applying a one-at-atime variation in the ionic currents through the sarcolemma and the SR membrane. Specifically, each current was multiplied by 0 (complete block), 0.3, 0.7, 1 (no change) and 2 in order to study their impact on selected calciumrelated biomarkers. These changes could represent the effect of drugs or mutations, or simply inter-individual physiological variability [14]. Simulations were performed at three different pacing rates $(1 \mathrm{~Hz}, 2 \mathrm{~Hz}$ and $3 \mathrm{~Hz})$ and ran until achieving steady-state. The measured calcium 
biomarkers include systolic $(\mathrm{CaS})$ and diastolic $(\mathrm{CaD})$ intracellular $\mathrm{Ca}^{2+}$ concentrations, $\mathrm{Ca}^{2+}$ transient duration at $30 \%$ recovery (CaTD30) and at $90 \%$ recovery (CaTD90), and maximum upstroke calcium velocity (CaDotMax).

The sensitivity between the most severe current variation and the "no change" condition was calculated as explained in [10]. Finally, sensitivities were normalized to the maximum absolute sensitivity for each biomarker [10].

\section{Results and Discussion}

Figs. 1 and 2 show representative examples of the results of the simulations for the two main ionic currents found to exert the stronger influence on the calcium biomarkers: $\mathrm{I}_{\mathrm{NaK}}\left(\mathrm{Na}^{+}-\mathrm{K}^{+}\right.$pump, Fig. 1) and $\mathrm{I}_{\text {up }}$ (SERCA pump, Fig. 2). The upper panels in these figures show action potential traces, while the lower panels depict intracellular calcium transients. The three curves in each panel represent a control simulation (black solid lines), a simulation in which the corresponding ionic current was partially blocked (x0.7, red dashed lines) and a third one in which the current was potentiated (x2, blue dotted lines).

The results seen in the figures indicate that, generally speaking, the variations in the ionic currents have a stronger impact in the intracellular calcium transients than in the action potential itself. Blocking the $\mathrm{Na}^{+}-\mathrm{K}^{+}$pump by $30 \%$, for instance, reduces the AP duration (APD) only slightly but increases the maximum systolic calcium concentration $(\mathrm{CaS})$ by $219 \%$. Similarly, if the pump current is doubled, APD is slightly increased while $\mathrm{CaS}$ decreases to $71 \%$ of its control value. A similar effect is also seen when the SERCA current is varied (Fig. 2).

Variations in other ionic currents affect the calcium biomarkers in a lesser extent and in different fashions. The case of $\mathrm{I}_{\mathrm{Kr}}$ (not shown) was of particular interest: variations in this current, which is a usual pharmacological target, changed APD to a significant extent but had almost no effect in calcium biomarkers.

The results obtained in the sensitivity analysis were then compiled and represented in Fig. 3 for two different pacing frequencies $(1 \mathrm{~Hz}$, corresponding to a basic cycle length (BCL) of $1000 \mathrm{~ms}$, and $3 \mathrm{~Hz}$, corresponding to a BCL of $333 \mathrm{~ms})$. Vertical bars represent the values of the biomarkers, normalized to their control values (100\%), as a function of the ionic current multiplying factor. Each biomarker is represented by a particular colour. Only the results for the SERCA current $\left(\mathrm{I}_{\mathrm{up}}\right)$ are shown, though similar graphs were built for each ionic current. The figure highlights the effect of pacing frequency on the influence of the SERCA pump in the intracellular calcium dynamics. A complete block of $I_{\text {up }}$, for instance, has a more pronounced effect on $\mathrm{CaD}$ at $3 \mathrm{~Hz}$ (237\% increase) than at $1 \mathrm{~Hz}(165 \%)$, conversely to the influence on $\mathrm{CaS}$ ( $72 \%$ and $100 \%$, respectively).

Finally, the results mentioned above were normalized and expressed as relative sensitivities, as explained in [10].

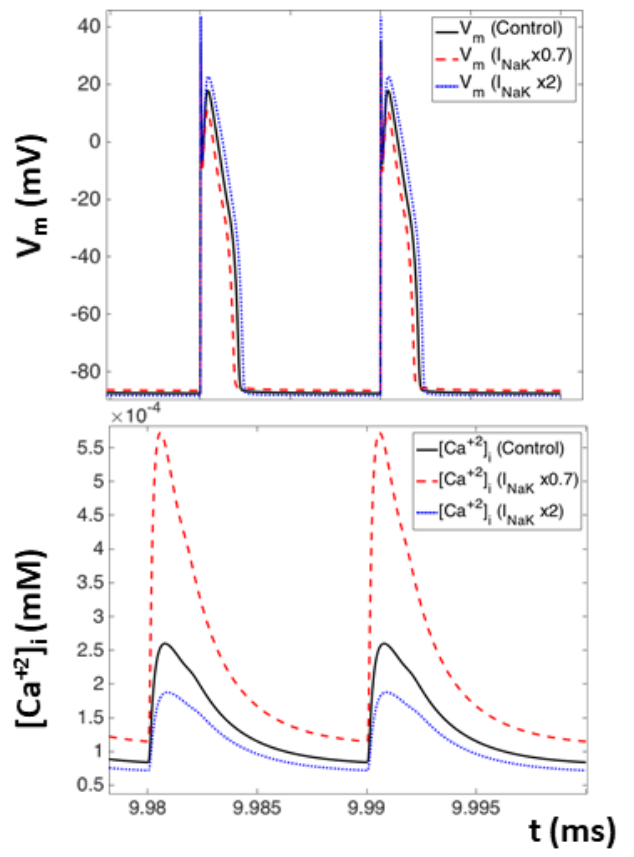

Figure 1. Action potentials (upper panel) and intracellular calcium transients (lower panel) for the control simulation (black traces), 30\% $\mathrm{I}_{\mathrm{NaK}}$ block (red) and $100 \% \mathrm{I}_{\mathrm{NaK}}$ potentiation (blue).
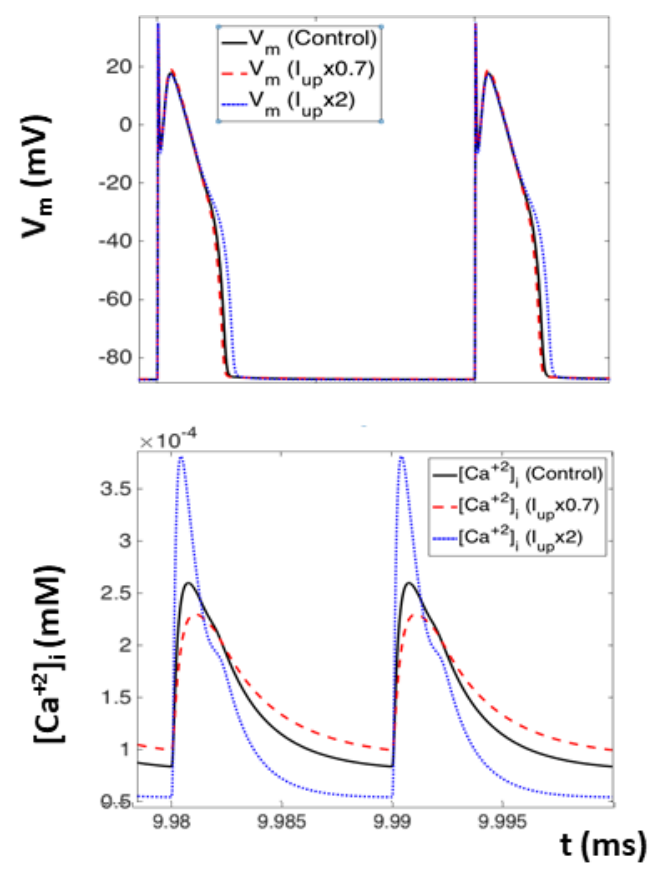

Figure 2. Action potentials (upper panel) and intracellular calcium transients (lower panel) for the control simulation (black traces), 30\% $\mathrm{I}_{\mathrm{SERCA}}$ block (red) and $100 \%$ ISERCA potentiation (blue). 

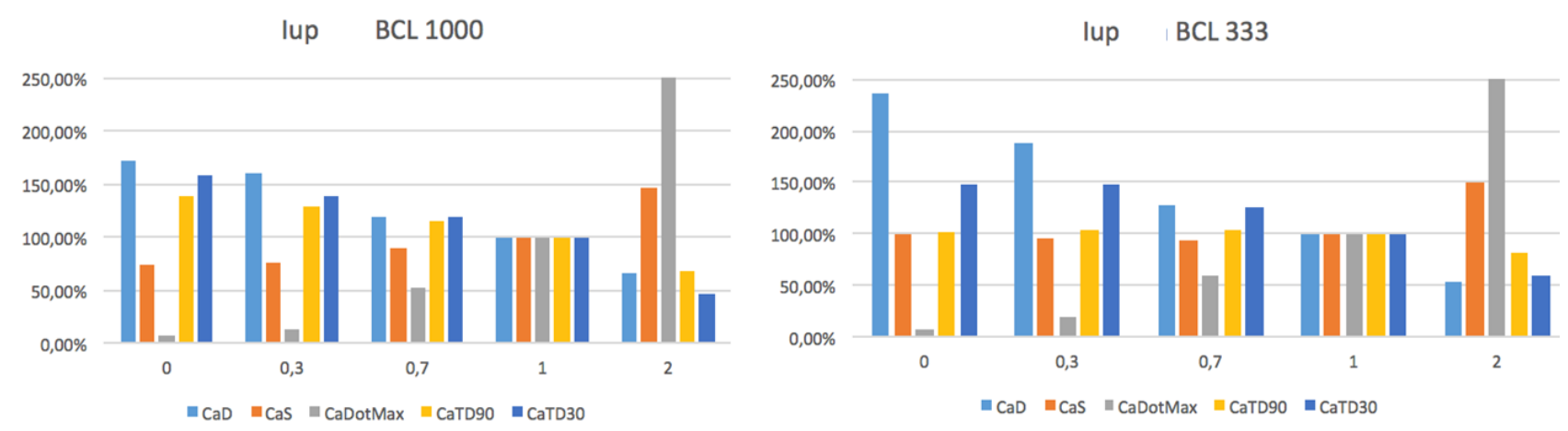

Figure 3. Compiled results of the sensitivity analysis for SERCA $\left(\mathrm{I}_{\mathrm{up}}\right)$ block $(\mathrm{x} 0, \mathrm{x} 0.3)$, control $(\mathrm{x} 1)$ and potentiation $(\mathrm{x} 2)$ for two different pacing frequencies. Bars show the relative value of each calcium biomarker.

Table 1. Relative sensitivities of the calcium biomarkers to changes in ionic currents for a pacing frequency of $1 \mathrm{~Hz}$.

\begin{tabular}{|c|c|c|c|c|c|c|c|c|c|c|c|c|c|}
\hline \multicolumn{14}{|c|}{ BCL 1000} \\
\hline $\mathrm{Rc}, \mathrm{p}$ & $\mathbf{I K r}$ & IKs & IK1 & INa & INaL & INaK & INaCa_cai & INaCa_sS & ICaL & IpCa & Irel & lup & Ito1 \\
\hline CaD & 0,028 & 0,002 & 0,031 & 0,038 & 0,024 & 0,953 & 0,318 & 0,304 & 0,349 & 0,068 & 0,054 & 1,000 & 0,013 \\
\hline CaS & 0,025 & 0,002 & 0,031 & 0,023 & 0,017 & 1,000 & 0,178 & 0,220 & 0,422 & 0,046 & 0,017 & 0,393 & 0,011 \\
\hline CaDotMax & 0,020 & 0,002 & 0,026 & 0,017 & 0,014 & 1,000 & 0,160 & 0,187 & 0,370 & 0,038 & 0,035 & 0,827 & 0,019 \\
\hline CaTD90 & 0,189 & 0,038 & 0,071 & 0,009 & 0,123 & 0,433 & 0,033 & 0,056 & 0,320 & 0,012 & 0,069 & 1,000 & 0,007 \\
\hline CaTD30 & 0,378 & 0,030 & 0,111 & 0,043 & 0,194 & 0,575 & 0,074 & 0,087 & 0,486 & 0,003 & 0,110 & 1,000 & 0,065 \\
\hline
\end{tabular}

Table 2. Relative sensitivities of the calcium biomarkers to changes in ionic currents for a pacing frequency of $2 \mathrm{~Hz}$.

\begin{tabular}{|c|c|c|c|c|c|c|c|c|c|c|c|c|c|}
\hline \multicolumn{14}{|c|}{ BCL 500} \\
\hline $\mathrm{Rc}, \mathrm{p}$ & $\mathbf{I K r}$ & IKs & IK1 & $\mathbf{I N a}$ & INaL & INaK & INaCa_cai & INaCa_SS & ICaL & IpCa & Irel & lup & Ito1 \\
\hline $\mathrm{CaD}$ & 0,009 & 0,001 & 0,034 & 0,061 & 0,037 & 0,880 & 0,220 & 0,219 & 0,437 & 0,056 & 0,037 & 1,000 & 0,003 \\
\hline CaS & 0,017 & 0,001 & 0,030 & 0,032 & 0,018 & 1,000 & 0,084 & 0,121 & 0,541 & 0,030 & 0,010 & 0,148 & 0,004 \\
\hline CaDotMax & 0,014 & 0,001 & 0,025 & 0,025 & 0,015 & 1,000 & 0,073 & 0,103 & 0,559 & 0,025 & 0,016 & 0,373 & 0,008 \\
\hline CaTD90 & 0,101 & 0,002 & 0,089 & 0,084 & 0,123 & 0,869 & 0,024 & 0,077 & 0,728 & 0,020 & 0,050 & 1,000 & 0,048 \\
\hline CaTD30 & 0,120 & 0,008 & 0,061 & 0,053 & 0,038 & 0,900 & 0,190 & 0,238 & 0,786 & 0,050 & 0,111 & 1,000 & 0,042 \\
\hline
\end{tabular}

Table 3. Relative sensitivities of the calcium biomarkers to changes in ionic currents for a pacing frequency of $3 \mathrm{~Hz}$.

\begin{tabular}{|c|c|c|c|c|c|c|c|c|c|c|c|c|c|}
\hline \multicolumn{14}{|c|}{ BCL 333} \\
\hline $\mathrm{Rc}, \mathrm{p}$ & $\mathbf{I K r}$ & IKs & IK1 & $\mathrm{INa}$ & INaL & INaK & INaCa_cai & INaCa_ss & ICaL & IpCa & Irel & lup & Ito1 \\
\hline $\mathrm{CaD}$ & 0,014 & 0,001 & 0,043 & 0,071 & 0,029 & 1,000 & 0,131 & 0,157 & 0,568 & 0,047 & 0,020 & 0,663 & 0,018 \\
\hline CaS & 0,038 & 0,002 & 0,059 & 0,065 & 0,021 & 1,000 & 0,082 & 0,144 & 0,792 & 0,040 & 0,011 & 0,194 & 0,011 \\
\hline CaDotMax & 0,010 & 0,001 & 0,015 & 0,015 & 0,004 & 1,000 & 0,020 & 0,034 & 0,412 & 0,010 & 0,005 & 0,114 & 0,003 \\
\hline CaTD90 & 0,250 & 0,040 & 0,191 & 0,072 & 0,057 & 0,919 & 0,070 & 0,143 & 0,933 & 0,012 & 0,116 & 1,000 & 0,033 \\
\hline CaTD30 & 0,010 & 0,001 & 0,022 & 0,070 & 0,048 & 1,000 & 0,205 & 0,308 & 0,749 & 0,063 & 0,041 & 0,879 & 0,0003 \\
\hline
\end{tabular}

For each biomarker, the current whose variations most affect the value of the biomarker is the 1.0 reference, so the effect of the variations of the other currents receive a value between 0 and 1 . The results are compiled in Table 1 (for $1 \mathrm{~Hz}, \mathrm{BCL}=1000 \mathrm{~ms})$, Table $2(2 \mathrm{~Hz}, \mathrm{BCL}=500 \mathrm{~ms})$ and Table $3(3 \mathrm{~Hz}, \mathrm{BCL}=333 \mathrm{~ms})$. Bold numbers indicate a direct relationship (i.e. the biomarker increases with the multiplying factor), while italic numbers indicate and inverse relationship. Dark orange color indicates the strongest sensitivity per row (value equal to 1 ), while mild orange corresponds to a lack of dependency between biomarker and multiplying factor (value equal to 0 ).

The results shown in the tables indicate that the $\mathrm{Na}^{+} / \mathrm{K}^{+}$ pump $\left(\mathrm{I}_{\mathrm{NaK}}\right)$, the current through the L-type $\mathrm{Ca}^{2+}$ channels
( $\left.\mathrm{I}_{\mathrm{CaL}}\right)$ and the SERCA pump $\left(\mathrm{I}_{\mathrm{up}}\right)$ are the three currents that have more impact on the calcium biomarkers. Surprisingly, the $\mathrm{Na}^{+} / \mathrm{Ca}^{2+}$ exchanger only exerts a mild influence on calcium dynamics (which is in accordance with recent experimental results [15]), the same as the RyR release current in the SR $\left(\mathrm{I}_{\mathrm{rel}}\right)$. The rest of the sarcolemmal currents play a negligible role.

\section{Conclusion}

According to our simulation results, the ionic currents that exert a stronger influence on intracellular calcium transients during the canine ventricular cardiac action 
potential are the $\mathrm{Na}^{+} / \mathrm{K}^{+}$pump, the current through the $\mathrm{L}$ type $\mathrm{Ca}^{2+}$ channels and the SERCA pump, with the other sarcolemmal and SR currents playing a minor role.

\section{Acknowledgements}

This work was partially supported by Dirección General de Política Científica de la Generalitat Valenciana (PROMETEO 2016/088), and by the "Plan Estatal de Investigación Científica y Técnica y de Innovación 20132016" from the Ministerio de Economía, Industria y Competitividad of Spain (DPI2016-75799-R) and AEI/FEDER, UE.

\section{References}

[1] Eisner DA, Caldwell JL, Kistamás K, Trafford AW. Calcium and Excitation-Contraction Coupling in the Heart. Circ Res. 2017 Jul 7;121(2):181-195.

[2] Ter Keurs HEDJ, Boyden PA. Calcium and Arrhythmogenesis. Physiol Rev. 2007 Apr; 87(2): 457506.

[3] Piacentino V, Weber CR, Chen X, Weisser-Thomas J, Margulies KB, Bers DM, et al. Cellular basis of abnormal calcium transients of failing human ventricular myocytes. Circ Res. 2003;92(6):651-8.

[4] Bers DM, Shannon TR. Calcium Movements Inside the Sarcoplasmic Reticulum of Cardiac Myocytes. J Mol Cell Cardiol. 2013 May; 58: 59-66.

[5] Trénor B, Romero L, Cardona K, Gomis J, Saiz J, Ferrero JM. Multiscale Modeling of Myocardial Electrical Activity: From Cell to Organ. Applied Biomedical Engineering, Dr. Gaetano Gargiulo (Ed.), InTech, DOI: $10.5772 / 22907$.

[6] Luo CH, Rudy Y. A model of the ventricular cardiac action potential. Depolarization, repolarization, and their interaction. Circ Res. 1991 Jun;68(6):1501-26.

[7] Decker KF, Heijman J, Silva JR, Hund TJ, Rudy Y. Properties and ionic mechanisms of action potential adaptation, restitution, and accommodation in canine epicardium. 1D simulation study. Am J Physiol Heart Circ Physiol. 2009 Apr;296(4):H1017-26.

[8] Shannon TR, Wang F, Puglisi J, Weber C, Bers DM. A mathematical treatment of integrated $\mathrm{Ca}$ dynamics within the ventricular myocyte. Biophys J. 2004 Nov;87(5):335171.

[9] O’Hara T, Virág L, Varró A, Rudy Y. Simulation of the undiseased human cardiac ventricular action potential: Model formulation and experimental validation. PLoS Comput Biol. 2011;7(5).

[10] Romero L, Carbonell B, Trenor B, Rodríguez B, Saiz J, Ferrero JM. Systematic characterization of the ionic basis of rabbit cellular electrophysiology using two ventricular models. Prog Biophys Mol Biol. 2011 Oct;107(1):60-73.

[11] Gomez JF, Cardona K, Romero L, Ferrero JM, Trenor B. Electrophysiological and structural remodeling in heart failure modulate arrhythmogenesis. 1D simulation study. PLoS One. 2014;9(9).

[12] Antzelevitch C, Belardinelli L et al. Electrophysiologic Effects of Ranolazine. A Novel Anti-Anginal Agent with Antiarrhythmic Properties. Circulation. 2004 Aug 24; 110(8): 904-910.

[13] L Virág, N Jost, R Papp, I Koncz, A Kristóf, Z Kohajda, G Harmati, B Carbonell-Pascual, JM Ferrero Jr, JG Papp, PP Nánási, and A Varró. Analysis of the contribution of Ito to repolarization in canine ventricular myocardium. $\mathrm{Br} \mathrm{J}$ Pharmacol. 2011 Sep; 164(1): 93-105.

[14] Muszkiewicz A, Britton OJ, Gemmell P, Passini E, Sánchez C, Zhou X, Carusi A, Quinn TA, Burrage K, Bueno-Orovio A, Rodriguez B. Variability in cardiac electrophysiology: Using experimentally-calibrated populations of models to move beyond the single virtual physiological human paradigm. Prog Biophys Mol Biol. 2016 Jan;120(1-3):115-27.

[15] Kohajda Z, et al. The Effect of a Novel Highly Selective Inhibitor of the Sodium/Calcium Exchanger (NCX) on Cardiac Arrhythmias in In Vitro and In Vivo Experiments. PLoS One. 2016; 11(11): e0166041.

Address for correspondence.

Chema Ferrero

Centro de Investigación e Innovación en Bioingeniería (Ci2B)

Universitat Politècnica de València

Camino de Vera s/n Valencia 46022, Spain

cferrero@ci2b.upv.es 